\title{
Analysis of morphology of crystals based on identification of interfacial structure
}

\author{
X. Y. Liu \\ RIM, Department of Solid State Chemistry, Faculty of Science, University of Nijmegen, Toernooivled 1, \\ 6525 ED Mijmegen, The Netherlands \\ E. S. Boek \\ Schlumberger Cambridge Research, High Cross, Madingley Road, Cambridge CB3 OEL, England \\ W. J. Briels \\ Chemical Physics Laboratory, University of Twente, P.O. Box 217, 7500 AE Enschede, The Netherlands \\ P. Bennema \\ RIM, Department of Solid State Chemistry, Faculty of Science, University of Nijmegen, Toernooivled 1, \\ 6525 ED Nijmegen, The Netherlands
}

(Received 13 February 1995; accepted 24 May 1995)

\begin{abstract}
A new theoretical approach for the prediction of the growth habit of crystals is presented. This approach is based on a newly derived relation between the growth rate of crystal surfaces and habit-controlling factors, and includes a key step: a so-called interface structure (IS) analysis. This analysis is to formulate the influence of the fluid phase on the crystal morphology. The essential of the IS analysis is to identify the adsorbed growth units which is in dynamic equilibrium with solid units at the crystal surface, and to calculate their concentration. It follows that a key external habit-controlling factor, the so-called surface scaling factor, can be calculated from the analysis. Based on detailed molecular dynamic (MD) simulation data, our formalism is applied to predict the morphology of urea crystals grown from aqueous solutions. Urea crystals grown from the solutions turn out to possess a needlelike shape, in excellent agreement with experiments. This is one of the first examples of the successful theoretical prediction of morphology of crystals, and will provide a new way of thinking and understanding of the influence of the mother phase on crystal habits. () 1995 American Institute of Physics.
\end{abstract}

\section{INTRODUCTION}

The problem related to why a particular crystal grown from a certain environment possesses a certain shape has drawn much attention for centuries. ${ }^{1}$ Recently, research on the influence of tailor-made additives on the morphology of crystals becomes increasingly important for industries. The shape of a crystal may be described by the distances from the center of the crystal to the respective crystal faces $\{h k l\} .{ }^{1-3}$ For a growing crystal these distances are proportional to the relative growth rates $R_{h k l}^{\text {rel }}$ of the crystal faces. The growth of crystal faces is governed by the growth kinetics. From the point of view of growth kinetics, the growth rate is not only dependent on the crystal structure, but also is drastically influenced by crystal growth conditions and the ambient phase. ${ }^{4-7}$ In order to predict the growth morphology of crystals, one needs to define properly habit-controlling factors and to relate the relative growth rate of the crystal face $(h k l)$ with these habit-controlling factors.

In the past, some traditional ad hoc theories ${ }^{2-3}$ have been published to predict the growth morphology of crystals. Among those, the Hartman-Perdok theory ${ }^{3}$ is the one which is used quite frequently. According to this theory, the relative growth rate of crystal faces $\{h k l\}$ is taken to be in direct proportion to the attachment energy of the faces $E_{h k l}^{\text {att }}\left(E_{h k l}^{\text {att }}\right.$ is the energy released per structural unit when a slice of crystal in the orientations $\{h k l\}$ is attached to the crystal surface. ${ }^{3}$ ) Applying this recipe implies that the morphology of crystals grown from solutions or the melt is the same as the vacuum morphology. ${ }^{3-5}$ In comparison with experiments, it was found ${ }^{6}$ that this theory works only when crystals are grown from the vapor phase. In case crystals are grown from solutions or the melt, discrepancies between the theoretical and the observed morphology occur frequently. Obviously, this is attributed to two reasons. First, the relation between the relative growth rate and habit-controlling factors has not been logically established. Second, the influence of the ambient phase on the morphology of crystals is not sufficiently taken into account.

In this paper, our attention will be concentrated on the second issue: the influence of the fluid phase on the growth morphology of crystals. We notice that the crystal-fluid interfaces are boundaries between the crystal phase and the ambient phase (including solute, solvents and impurities). ${ }^{8}$ The structure of these regions has a strong impact on the growth kinetics, ${ }^{8-15}$ and on the growth rate and the morphology of crystals. For a given crystal surface, this structure is directly altered by the ambient phase and the condition of crystal growth. ${ }^{9-15}$ Therefore, we will formalize the influence of the ambient phase by analyzing the structure of the crystal-fluid interface. In correspondence to the periodic bond chain (PBC) analysis, ${ }^{3-7}$ which is conventionally used to examine the influence of the crystal structure on the morphology of crystals, this analysis will be called hereafter the interfacial structure (IS) analysis. In this paper, a basic relation between the growth rate and habit-controlling factors is derived first and the main principles and procedures of the IS analysis will be presented. Our formalism is applied to pre- 
dict the growth morphology of urea crystals grown from aqueous solutions.

\section{GROWTH HABIT AND INTERFACIAL STRUCTURE ANALYSIS}

\section{A. Solid-fluid interfaces and relative growth rate of crystal faces}

The growth of crystals bounded by facets is usually governed by the spiral mechanism. ${ }^{6,7}$ This means that a smooth crystal surface may contain one or more screw dislocations, providing the surface with spiral steps. Growth takes place by the deposition and incorporation of solute molecules from the bulk into these steps at a certain supersaturation $\Delta \mu / k_{b} T$, causing them to advance with a speed $V_{\text {step }}$ along the surface, perpendicularly to the step. $\left(\Delta \mu=\mu^{f}-\mu^{s}, \mu^{f}\right.$ is the chemical potential of a solute molecule and $\mu^{s}$ of the solid molecule.) Assuming that the average distance between two steps is $\lambda_{h k l}$ and their heights are $d_{h k l}$, the growth rate of the surface is then given by $R_{h k l}=V_{\text {step }} d_{h k l} / \lambda_{h k l} \cdot{ }^{6,7}$ Here, the shape of $V_{\text {step }}$ depends on the way of delivery of growth units to the steps. ${ }^{6}$ For the growth of crystals from a solution, the surface diffusion of solute molecules to steps is irrelevant, because the mean square displacement of an adsorbed solute molecule at the surface is short. ${ }^{6}$ Solute molecules are delivered directly from the bulk to steps at the surface via the volume diffusion. This includes two major steps: (i) the delivery of growing substances to the steps by means of volume diffusion; (ii) the integration of growth molecules into the crystal at kinks. At relatively low supersaturations, (ii) is the rate determining step, and the rate is proportional to the concentration of adsorbed solute molecules $X_{A(h k l)}$ around the kinks, the kink density $\rho_{h k l}^{\mathrm{kink}}$ and $\exp \left\{-\Delta G_{h k l}^{\neq} l k_{b} T\right\}$. Therefore, $V_{\text {step }} \propto X_{A(h k l)} \rho_{h k l}^{\text {kink }} \exp \left\{-\Delta G_{h k l}^{\neq} / k_{b} T\right\}$ for a given $\left(\Delta \mu / k_{b} T\right){ }^{6,7}$ It follows that the relative growth rate of crystal faces $\{h k l\}$

$$
R_{h k l}^{\mathrm{rel}} \propto \frac{d_{h k l}}{\lambda_{h k l}} \rho_{h k l}^{\mathrm{kink}} X_{A(h k l)} \exp \left\{-\Delta G_{h k l}^{\neq} / k_{b} T\right\},
$$

where $X_{A(h k l)}$ is the solute concentration at the face $(h k l)$, and $\Delta G_{h k l}^{\neq}$is the activation free energy for adsorbed solute molecules to be incorporated into kinks. In order to relate the relative growth rate $R_{h k l}^{\text {rel }}$ to quantities which can be easily obtained from a molecular dynamics simulation, we need to introduce the concept of effective growth units. The so-called effective growth units are those solute molecules adsorbed at the surface which are active in dynamic equilibrium with the solid molecules at the kinks. These molecules include the adsorbed solute molecules which are properly oriented in reference to the solute units at the surface and those which cost almost no energy to be transformed to the proper orientation (cf. Sec. II B).

Let us now analyze Eq. (1) in some detail. In order to be incorporated into the kinks, adsorbed solute molecules should have a proper orientation with respect to the solid molecules at the surface. ${ }^{6,8}$ According to the definition of effective growth units, the probability for adsorbed solute molecules to obtain the proper orientation is about $X_{A(h k l)}^{\text {eff }} / X_{A(h k l)}$. $\left(X_{A(h k l)}^{\text {eff }}\right.$ denotes the concentration of effective growth units at the surface.) Therefore, $\exp \left\{-\Delta G_{h k l}^{ \pm} / k_{b} T\right\}$ $\approx\left(X_{A(h k l)}^{\text {eff }} / X_{A(h k l)}\right) \exp \left\{-\Delta G_{\text {kink }}^{*} / k_{b} T\right\} . \Delta G_{\text {kink }}^{*}$, the desolvatation activation energy, is supposed to be independent of the specific surface, provided that the interactions between solid and solvent units at the surface is weak. ${ }^{3,6,9}$

In the case of the spiral growth, the spiral is rotating with constant angular velocity, guided by the screw dislocation center. Some distance from the center, the spiral becomes roughly equal to concentric circular steps with a distance $\lambda_{h k l} \cong 19 r_{c} \cdot{ }^{6,7}$ Here, $r_{c}$ is the radius of the two-dimensional critical nucleus given by $r_{c} \approx \tilde{\phi}_{\text {step }} \Omega / \Delta \mu,{ }^{6,7} \tilde{\phi}_{\text {step }}$ is the average step energy of a step per growth unit and $\Omega$ is the molecular volume. Therefore, $\lambda_{h k l} \propto \tilde{\phi}_{\text {step }}$ at a given $\Delta \mu / k_{b} T$. $\rho_{h k l}^{\text {kink }} \sim \exp \left\{-\tilde{\phi}_{h k l}^{\text {kink }} / k_{b} T\right){ }^{6,7}$ Because the average kink energy $\tilde{\phi}_{h k l}^{\text {kink }} \approx \tilde{\phi}_{h k l}^{\text {step }}, \rho_{h k l}^{\text {kink }} \sim \exp \left\{-\tilde{\phi}_{h k l}^{\text {step }} / k_{b} T\right)$. Combining the results so far, we find

$$
R_{h k l}^{\mathrm{rel}} \propto \frac{d_{h k l} X_{A(h k l)}^{\mathrm{eff}}}{\tilde{\phi}_{h k l}^{\mathrm{step}}} \exp \left\{-\tilde{\phi}_{h k l}^{\mathrm{step}} / k_{b} T\right\} .
$$

Obviously, to find the expression of $\tilde{\phi}_{h k l}^{\text {step }}$ is one of the key issues. In the following, we shall arrive at

$$
\tilde{\phi}_{h k l}^{\mathrm{step}} \approx \xi_{h k l} C_{/(h k l)}^{*} \Delta H^{\mathrm{diss}} / n_{h k l} .
$$

Here, $n_{h k l}$ is the coordination number, i.e., the number of neighbors of a solid molecule within the $2 \mathrm{D}$ crystal slice $(h k l)$, and $\Delta H^{\text {diss }}$ is the experimentally observed enthalpy of dissolution. The crystallographic orientation factor $\xi_{h k l}$ and the surface scaling factor $C_{/(h k l)}^{*}$ will be defined below.

The step energy, the (1D) energy cost to create a step with the length of one molecule, can be related to the (3D) local dissolution enthalpy $\Delta H_{h k l}^{\text {diss }}$ at the crystal surface, ${ }^{7-9}$ which is the enthalpy change due to transforming a solid molecule at a kink to an adsorbed solute molecule. We may reduce $\Delta H_{h k l}^{\text {diss }}$ to a sum of in-plane contributions by multiplying it by $\xi_{h k l}=E_{h k l}^{\text {slice }} / E^{\text {cr }}$, where $E^{\text {cr }}$ is the lattice energy per molecule and $E_{h k l}^{\text {slice }}$ is the 2D lattice energy per molecule for a crystal slice $(h k l)$ with thickness $d_{h k l} .{ }^{3}$ Obviously, we may approximate ${ }^{16}$

$$
\tilde{\phi}_{h k l}^{\text {step }} n_{h k l} \approx \xi_{h k l} \times \Delta H_{h k l}^{\text {diss }} .
$$

We notice that because of the ordering of fluid molecules at the interface and the crystal relaxation near the surface, $\Delta H_{h k l}^{\text {diss }}$ is normally different from $\Delta H^{\text {diss }} \cdot{ }^{12}$ Nevertheless, we may still easily estimate $\Delta H_{h k l}^{\text {diss }}$ in terms of $C_{\ell(h k l)}^{*}$. The surface scaling factor $C_{/(h k l)}^{*}$ is defined ${ }^{12}$ as

$$
C_{\ell(h k l)}^{*}=\Delta H_{h k l}^{\mathrm{diss}} / \Delta H^{\mathrm{diss}} .
$$

From the van't Hoff relation, ${ }^{14}$ we have $\ln X_{A}=-\Delta H^{\text {diss }}$ $k_{b} T+\Delta H^{m} / k_{b} T^{m} \approx \Delta H^{\text {diss }}\left(T-T^{m}\right) / k_{b} T T^{m}$ ( $X_{A}$ is the concentration of solute molecules in the bulk, the superscript $m$ denotes melting). Applying the same theories to the dissolution process at the crystal surface, ${ }^{14}$ we have $\ln X_{A(h k l)}^{\mathrm{eff}}$ $=-\Delta H_{h k l}^{\mathrm{diss}} / k_{b} T-\Delta H_{h k l}^{m} / k_{b} T^{m} \approx \Delta H_{h k l}^{\mathrm{diss}}\left(T-T^{m}\right) / k_{b} T T^{m}$. From the above relations we get ${ }^{10}$

$$
C_{\ell(h k l)}^{*} \cong \ln X_{A(h k l)}^{\mathrm{eff}} / \ln X_{A} .
$$

From Eqs. (4), (5), and (6) we finally obtain Eq. (3). Substituting Eqs. (3) and (6) into Eq. (2) yields 


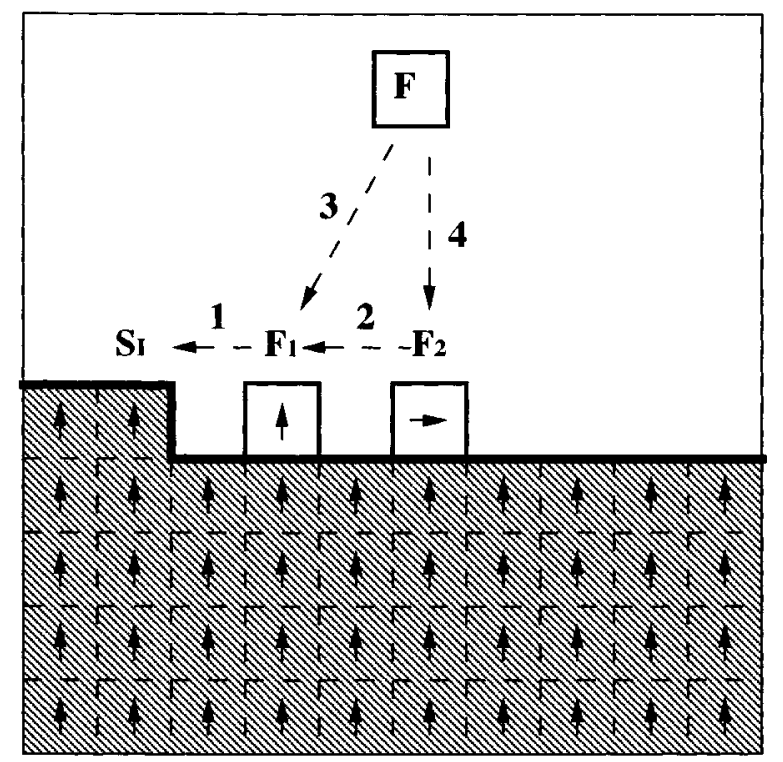

FIG. 1. A schematic illustration for the different states of structural units at the interfacial system and equilibria between different states.

$R_{h k l}^{\mathrm{rel}} \propto \frac{n_{h k l} d_{h k l}}{C_{l(h k l)}^{*} \xi_{h k l}} X_{A}^{C_{f(h k l)}^{*}} \exp \left\{-C_{l(h k l)}^{*} \xi_{h k l} \Delta H^{\mathrm{diss}} / n_{h k l} k_{b} T\right\}$.

Equation (7) is our basic formula for the prediction of growth morphologies of crystals. Note that $n_{h k l}, d_{h k l}$, and $\xi_{h k l}$ can easily be calculated from the crystal structure and a PBC analysis. ${ }^{3-5}$ The key issue then is to calculate $X_{A(h k l)}^{\text {eff }}$ from an analysis of the interfacial structure obtained from MD simulations.

\section{B. Interfacial structural (IS) analysis}

In order to calculate $C_{/(h k l)}^{*}$, one must know $X_{A(h k l)}^{\mathrm{eff}}$ for different crystal faces [see Eq. (6)]. The purpose of this analysis is to calculate $X_{A(h k l)}^{\text {eff }}$. The first step of the analysis is to identify the effective growth units. Then their concentration is calculated.

Let us first assume that the detailed structure of the solid-fluid interface is known. At the crystal surface, growth units can be roughly classified into two different types. Those with the orientations and the conformations similar to solid molecules (named $S_{\mathrm{I}}$ ) at the surface can be in any case directly incorporated into the solid phase at kink sites and are named $F_{1}$ units hereafter. (The kink sites are the sites with solid units of the highest energy level at the crystal surface. The growth and the dissolution of crystals will happen first at these sites.) The rest, which have to be transformed to $F_{1}$-like units before entering the crystal structure, are named $F_{2}$ units. These growth units of different states are schematically illustrated in Fig. 1 . According to the definition, $F_{1}$ units are in dynamic equilibrium with solid units $S_{\mathrm{I}}$ as

$$
F_{1} \stackrel{\text { I }}{\rightleftharpoons} S_{\text {I. }} \text {. }
$$

For $F_{2}$ units, the similar process can be expressed as

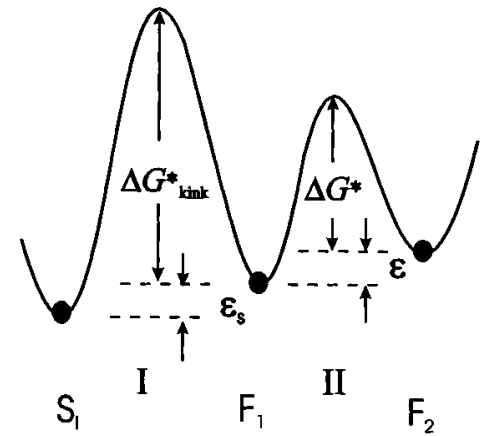

(a)

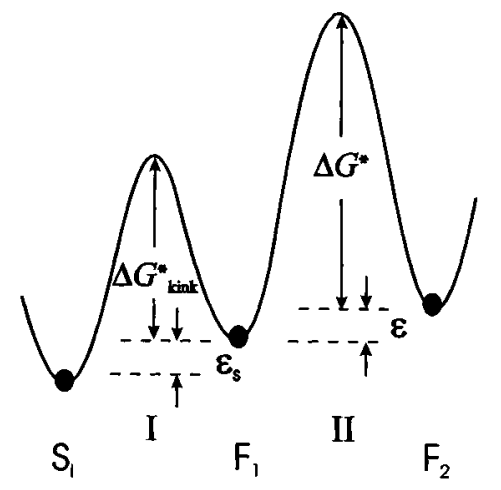

(b)

FIG. 2. Pictures of the potential landscape between different types of structural units at the surface. In equilibrium with interfacial crystal units $S_{\mathrm{I}}, F_{1}$ units change directly into $S_{\mathrm{I}}$ units by passing the potential barrier $\Delta G_{\text {kink }}^{*}$, while in a similar process, $F_{2}$ units should first change to $F_{1}$ then to $S_{\text {I }}$ units. ( $\Delta G^{*}$ is the potential barrier for the transition from $F_{2}$ to $F_{1}$.) It is shown in (a) that $\Delta G_{\text {kink }}^{*}>\Delta G^{*}$, implying $\zeta \approx \delta \approx 1$. In this case $F_{1}$ and $F_{2}$ units behave the same in the surface process. Both of them can be treated as the effective growth units. In (b), it is shown that $\Delta G_{\text {kink }}^{*}>\Delta G^{*}\left(R^{\mathrm{II}-\mathrm{I}} \ll R^{\mathrm{I}-\mathrm{S}}\right.$ and $\left.R^{\mathrm{II}-\mathrm{S}} \ll R^{\mathrm{I}-\mathrm{S}}\right)$. In this case only $F_{1}$ can be considered as the effective growth units $(\zeta \approx 0, \delta<1)$.

$$
F_{2} \stackrel{\text { II }}{\rightleftharpoons} F_{1} \stackrel{\text { I }}{\rightleftharpoons} S_{\text {I. }} .
$$

We notice that in spite of the processes I and II, there are other two processes, III and IV leading to the equilibrium between bulk fluid units $F$ and $F_{1}$ units and $F_{2}$ units (see Fig. 1). In contrast to I and II, the processes III and IV can be very complex processes depending on the growth kinetics. Nevertheless, we assume here that the chance for a certain type of structural units to be delivered to kink sites is independent off growth kinetics, and is equal to the mole fraction of the structural units at the surface. This implies that only the ordering of growth units at the surface are relevant for our discussions. Therefore, our major attention will be focused on the two processes indicated by Eqs. (8) and (9).

The different states of growth units at the surface are schematized in Fig. 2, and the potential barriers that must be overcome when a transition occurs between states are indicated. According to this figure, the rate for the transformation from a pure growth unit $F_{2}$ to a pure growth unit $F_{1}$ is

$$
R^{\mathrm{II}}=\nu_{2} \exp \left(-\Delta G^{*} / k_{b} T\right)
$$

and the probability for the reverse transition is

$$
\left.R_{-1}^{\mathrm{II}}=\nu_{1} \exp \left[\left(-\Delta G^{*}+\epsilon\right) / k_{b} T\right)\right]
$$


where $\nu_{1}$ and $\nu_{2}$ denote the frequency of thermal vibration for $F_{1}$ and $F_{2}$ units, respectively, $\Delta G^{*}$ is the potential barrier for the transition from $F_{2}$ to $F_{1}$, and

$$
\epsilon=\mu_{F_{2}}^{o}-\mu_{F_{1}}^{o}
$$

$\left(\mu_{k}^{o}\right.$ is the standard chemical potential of species $k$ ). Similarly, the rate for the transition from a pure $F_{1}$ unit to a pure solid unit at the surface (denoted by $S_{\mathrm{I}}$ ) and that for the reverse process can be expressed by

$$
R^{\mathrm{I}-\mathrm{S}}=\nu_{1} \exp \left(-\Delta G_{\text {kink }}^{*} / k_{b} T\right)
$$

and

$$
R_{-1}^{\mathrm{I}-\mathrm{S}}=\nu_{s} \exp \left[-\left(\Delta G_{\mathrm{kink}}^{*}+\epsilon_{s}\right) / k_{b} T\right],
$$

respectively. Here, $\Delta G_{\text {kink }}^{*}$ represents the potential barrier for the transition from a pure $F_{1}$ to an $S_{\mathrm{I}}$ unit, $\nu_{s}$ is the frequency of thermal vibration for $S_{\mathrm{I}}$ units and

$$
\epsilon_{s}=\mu_{S_{\mathrm{I}}}^{o}-\mu_{F_{1}}^{o}
$$

According to Eq. (9) and Fig. 2, the rate of the transition from a pure $F_{2}$ to an $S_{\mathrm{I}}$ turns out to be

$$
R^{\mathrm{II}-\mathrm{S}}=R^{\mathrm{II}} R^{\mathrm{I}-\mathrm{S}}\left(R^{\mathrm{II}}+R^{\mathrm{I}-\mathrm{S}}\right) .
$$

At equilibrium, the rate for the transition from a solid unit to a fluid unit is constant. However, the dynamic behavior for $F_{1}$ and $F_{2}$ units can be very much different. The question arises as to what extent an $F_{2}$ unit can be regarded as an effective growth units in equilibrium with $S_{\mathrm{I}}$ units. It follows from Eq. (15) that $R^{\mathrm{II}-\mathrm{S}}$ will be determined by the step (I or II) with the highest potential barrier. This implies that the rate of the transition from a pure $F_{2}$ unit to an $S_{\mathrm{I}}$ unit at a kink site will be equal to or lower than that of an $F_{1}$ unit. Taking the time scale of the transition from $F_{1}$ to $S_{\mathrm{I}}$ units as a reference, we define a factor $\zeta$ as an effective factor to characterize the effectivity of $F_{2}$ units. This factor is given as the following:

$$
\zeta=R^{\mathrm{II}-\mathrm{S}} / R^{\mathrm{I}-\mathrm{S}}=R^{\mathrm{II}} /\left(R^{\mathrm{II}}+R^{\mathrm{I}-\mathrm{S}}\right) .
$$

Following the definition, it can be seen that $0 \leqslant \zeta \leqslant 1$. That $\zeta=1$ implies that in dynamic equilibrium with $S_{\mathrm{I}}$ units, $F_{2}$ units behave the same as $F_{1}$ units. This occurs when growth units belong to simple and small molecules. (Obviously, $\zeta=1$ for $F_{1}$ units.) That $\zeta=0$ corresponds to the case that $F_{2}$ units are completely inactive in the process of the solid-fluid transition occurring at the surface. This means, in order to incorporate into the crystal structure, $F_{2}$-like growth units have to overcome an extremely high potential barrier (or the socalled "entropy barrier") to reorient themselves in the proper direction. Therefore, it becomes an almost impossible event. The growth of polyethylene crystals (or long chain paraffin crystals) in the $\{001\}$ orientations is a typical example, where some growth units with "wrong" conformations or configurations will block the growth of the crystal surface, resulting in the so-called "self-poisoning."

We notice that $F_{1}$ units need not be in a minimum free enthalpy state, and it may happens that there is only a very small fraction of $X_{A(h k l)}$ for a certain crystal face. However, there can still be some growth units which are oriented somewhat differently from $F_{1}$ molecules but still have $\zeta=1\left[\Delta G^{*}(\tau) \leqslant 0\right]$. We regard these growth units, together with the well-defined $F_{1}$ units, as $F_{1}$-like units. Others with $\Delta G^{*}>0$ are $F_{2}$-like growth units.

For the aforementioned case, we can define the concentration of effective growth units in the following way:

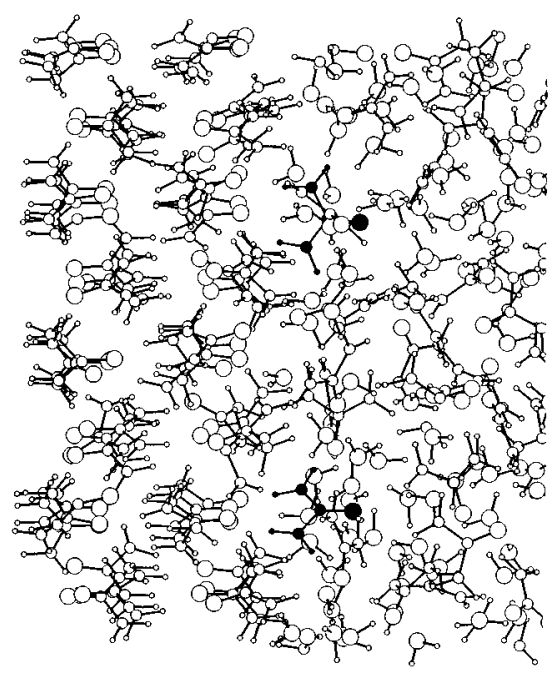

(a)
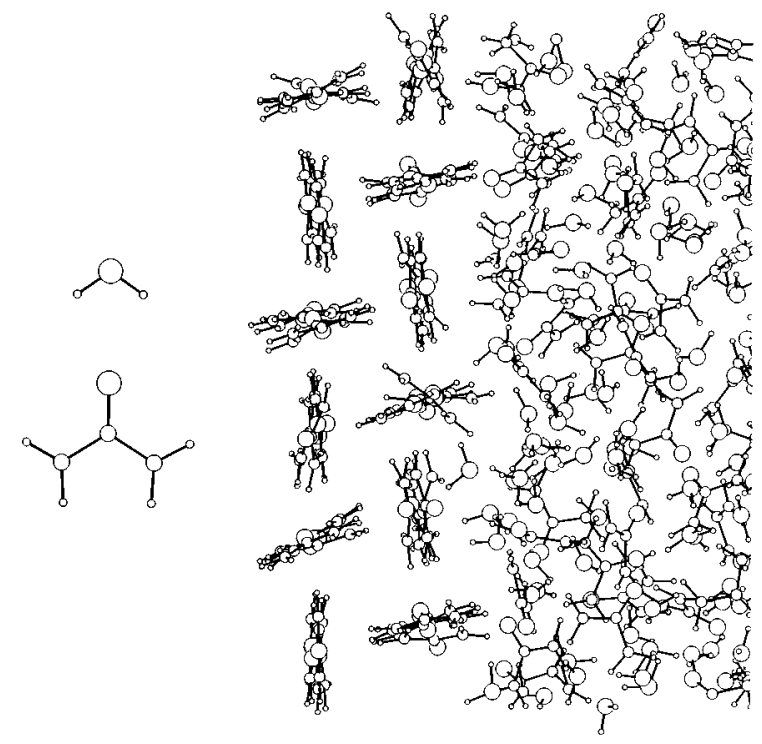

(b)

FIG. 3. Snapshots of interfaces between crystalline urea (left) and saturated aqueous urea solutions (right). In (a) the (001) interface is shown, in (b) the (110) interface is presented. In between, a water molecule (above) and a urea molecule (below) are drawn for clarification. These snapshots have been generated by means of molecular dynamics simulations (Refs. 18,19). 


$$
X_{A(h k l)}^{\mathrm{eff}}=\zeta X_{F 2}+X_{F 1}=\sum_{\tau=F_{1}, F_{2}}\left[\zeta(\tau) X_{A(h k l)}^{\tau}\right]=\delta X_{A(h k l)}
$$

where $X_{A(h k l)}=X_{F 2}+X_{F 1}=\Sigma_{\tau=F_{1}, F_{2}}\left[X_{A(h k l)}^{\tau}\right]$, and $X_{A(h k l)}^{\tau}$ is the concentration of adsorbed solute units at the conformational state $\tau, \delta$ is of a value between 0 and 1 . Introducing the factor $\delta$ implies that $X_{A(h k l)}^{\text {eff }}$ is only a fraction of $X_{A(h k l)}$. According to Eq. (17), $\delta$ can be generally expressed as

$$
\delta=\sum_{\tau=F_{1}, F_{2}}[\zeta(\tau) P(\tau)]
$$

with

$$
P(\tau)=X_{A(h k l)}^{\tau} / X_{A(h k l)} .
$$

Here, $P(\tau)$ denotes the probability for an adsorbed growth unit at the conformation state $\tau$. Assuming $\nu_{1}, \nu_{2}$, and $\nu_{s}$ are of the same magnitude, it follows from Eq. (17) that the two typical cases can be distinguished:

I. $\Delta G^{*} / k_{b} T \ll \Delta G_{\text {kink }}^{*} / k_{b} T$. This corresponds to $\zeta \approx 1$ (and $\delta \approx 1$ ), implying that both $F_{1}$ and $F_{2}$ are regarded as effective units and

$$
X_{A(h k l)}^{\mathrm{eff}} \approx X_{A(h k l)} \text {. }
$$

II. $\Delta G^{*} / k_{b} T \gg \Delta G_{\mathrm{kink}}^{*} / k_{b} T$. It follows that $\zeta \approx 0$, and

$$
X_{A(h k l)}^{\mathrm{eff}} \approx X_{F 1}<X_{A(h k l)}(\delta<1) .
$$

In this case, only $F_{1}$ units are considered to be active. In spite of this, if $\Delta G^{*}$ is comparable to or slightly larger than $\Delta G_{\text {kink }}^{*}, F_{2}$ units can be partly considered as effective growth units, and the value of $\zeta$ will occur between 1 and 0 .

Now it can be seen that to identify $F_{1}$ units and to calculate the density of the orientation distributions of all growth units at the interface will be a crucial step in this analysis. $F_{1}$ units can easily be recognized in reference to the crystal surface structure obtained from a PBC analysis. Furthermore, the density $X_{A(h k l)}$ and the probability of orientational distributions of growth units at the crystal surface should be calculated. These can be done by carrying out density functional theory calculations, Monte Carlo (MC), molecular dynamics (MD) computer simulations, selfconsistent field calculations or they can be obtained from experimental data, etc. ${ }^{11,12,15-19}$ From these data, we can calculate quantitatively the values of $\Delta G^{*}$ for various states of growth units at the surface, and therefore estimate $X_{A(h k l)}^{\mathrm{eff}}$.

We notice that, for normal cases, interfacial fluid units are distributed continuously in various conformational and configurational states instead of distributions in separated states. This makes the distinction between the aforementioned two cases a bit difficult. Nevertheless, the same principles can still be applied. We only need to calculate $\delta$ and then $X_{A(h k l)}^{\text {eff }}$ [see Eq. (17a)].

According to principles of statistical thermodynamics, ${ }^{14}$ $P(\tau)$ can be expressed as

$$
P(\tau) \sim \exp \left[-G^{*}(\tau) / k_{b} T\right]
$$

or

$$
G^{*}(\tau)=-k_{b} T \ln [P(\tau)]+\text { const. }
$$

It follows that the plot of $G^{*}(\tau)$ can be calculated from $P(\tau)$. $\Delta G^{*}(\tau)$ can then be also obtained from the $G^{*}(\tau)$ plot. Since $P(\tau)$ changes continuously, $\Delta G^{*}(\tau)$ changes also continuously with $\tau$. If $\Delta G_{\text {kink }}^{*}$ can be reasonably estimated, the concentration of effective growth units for face $(h k l)$ can be generally calculated according to Eqs. (16) and (17a) by integrating $\delta(\tau)$ at different states

$$
\begin{aligned}
& \delta= \int \zeta(\tau) P(\tau) d \tau=1 / 2 \int P(\tau) \\
& \times \exp \left\{\left[\Delta G_{\mathrm{kink}}^{*}-\Delta G^{* *}(\tau)\right] / 2 k_{b} T\right\} \\
& \times \operatorname{sech}\left\{\left[\Delta G_{\mathrm{kink}}^{*}-\Delta G^{* *}(\tau)\right] / 2 k_{b} T\right\} d \tau . \\
&\left(\Delta G^{* *}(\tau)=0 \quad \text { if } \quad \Delta G^{*}(\tau) \leqslant 0 ; \quad \Delta G^{* *}(\tau)=\Delta G^{*}(\tau) \quad\right. \text { if } \\
&\left.\Delta G^{*}(\tau)>0 .\right)
\end{aligned}
$$

\section{MORPHOLOGY OF UREA CRYSTALS GROWN FROM AQUEOUS SOLUTIONS}

Urea $\left[\mathrm{O}=\mathrm{C}\left(\mathrm{NH}_{2}\right)_{2}\right]$ crystallizes in the tetragonal space group $P_{42}{ }_{1} \mathrm{~m}(a=5.661 \AA ⿻, c=4.712 \AA) .{ }^{20}$ According to experiments, ${ }^{21,22}$ urea crystals grown from aqueous solutions are mainly bounded by the $\{001\},\{111\}$, and the $\{110\}$ faces. We will in the following ignore the $\{111\}$ faces for the sake of simplification. The factors $n_{h k l}, \xi_{h k l}$, and $d_{h k l}$ for the orientations can be obtained from a PBC analysis. ${ }^{23}$

As aforementioned, to characterize the influence of the fluid phase, we first carry out the IS analysis to evaluate $C_{\ell(h k l)}^{*}$. Then the growth morphology of urea crystals is constructed based on the calculated results.

The IS analysis is carried out based on the structure of the solid-fluid interface. Therefore as the first step of the analysis, the (fluid) interfacial structure of the urea-water solution system should be determined or calculated. Here, molecular dynamics simulations were carried out to compute the structure of solid-fluid interfaces.

The molecular dynamics simulations were performed with the GROMOS package. ${ }^{24}$ The computational boxes consisted of a saturated urea solution (with a urea mole fraction of 0.265 ) in contact with two different urea crystal surfaces. A constant $N V T$ equilibration of $10 \mathrm{ps}$ at high temperature (798 K) was performed to remove any spurious structure in the solution, followed by a $10 \mathrm{ps} N V T$ equilibration run at $298 \mathrm{~K}$. Then the solution was brought into contact with the crystal. In order to allow for volume relaxation, the crystalsolution box was equilibrated at constant, which destroys urea tetragonal crystal structure. Therefore, we continued at this point with a $20 \mathrm{ps} N V T$ equilibrium run at $298 \mathrm{~K}$, followed by $N V T$ production runs of $70 \mathrm{ps}$ for both interface. Using this technique, the Newtonian equations of motion are integrated numerically for a large number of particles. In our case, the computational box consists of 300 crystalline urea molecules, 200 solute urea molecules and 500 water molecules. In these snapshots only the interfacial region of the computational box is shown. Simulation runs of about $200 \mathrm{ps}$ have been performed for both interfaces. 


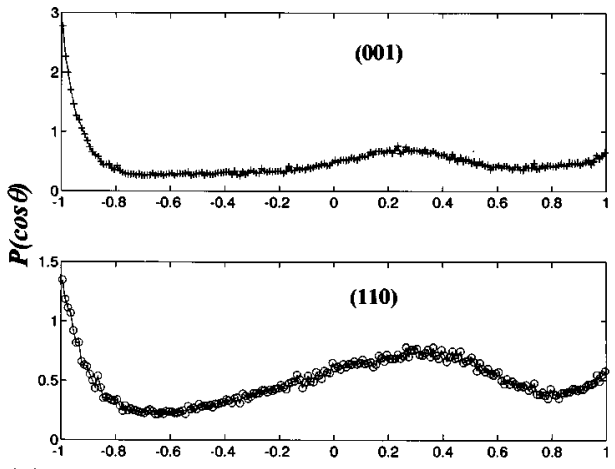

(a)

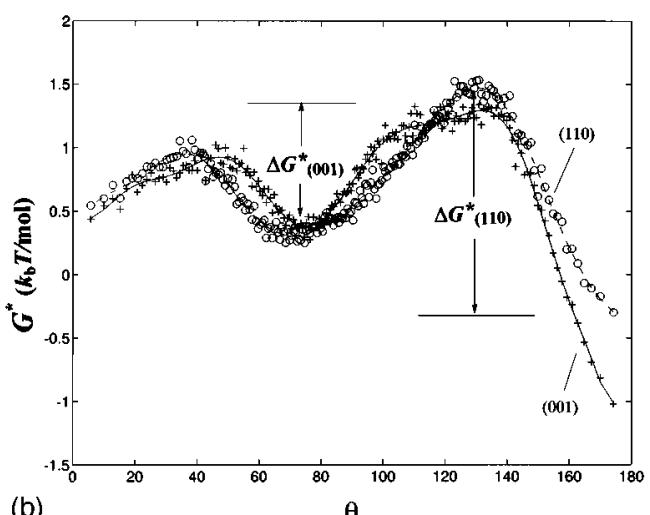

(b)

FIG. 4. (a) Orientational probability distributions of the dipole vectors of adsorbed solute urea molecules at the (001) interface and the (110) interface. The distributions have been calculated as a function of $\cos \theta(\theta$ is the angle between the dipole vectors and the outward surface normal) by averaging the time series of coordinates of the solute urea molecules in the first adsorbed liquid layer. (b) The potential of adsorbed urea molecules plotted versus orientations of the dipole vectors of the molecules at the (001) interface (indicated by + ) and the (110) interface (indicated by $\bigcirc$ ). The potential is calculated on the basis of Eq. (20a). Note that for the orientation of (001) a small minimum at $\theta \sim 0$ in our present calculations corresponds to the ordering of urea molecules occurring in the second fluid layer.

The force field for urea was constructed from the HHL potential $^{25}$ for nonbonded interactions and GROMOS covalent parameters. ${ }^{26}$ For water the SPC/E potential ${ }^{27}$ was used. Covalent bond distances were constrained using the SHAKE algorithm, ${ }^{28}$ which allows an integration time step of $2 \times 10^{-15}$ s. Coordinates were samples every 25 time steps. The total simulation time was approximately $650 \mathrm{CPU}$ hours on a Convex C240 vector computer. For more details about the simulations, we refer to Refs. 18 and 19.

TABLE I. Habit-controlling factors and relative growth rates for the $\{001\}$ and the $\{110\}$ faces of urea crystals.

\begin{tabular}{lccccc}
\hline \hline & $d_{h k l}(\AA)^{\mathrm{d}}$ & $n_{h k l}{ }^{\mathrm{a}}$ & $\xi_{h k l}{ }^{\mathrm{a}}$ & $C_{l(h k l)}^{*}{ }^{\mathrm{b}}$ & $R_{h k l}^{\text {rel }}$ \\
\hline$\{001\}$ & 4.71 & 2.00 & 0.56 & 0.96 & 34.90 \\
$\{110\}$ & 4.00 & 2.00 & 0.67 & 1.78 & 1.00 \\
\hline \hline
\end{tabular}

${ }^{\mathrm{a}} \xi_{h k l}$ and $n_{h k l}$ were calculated based on the results of the PBC analysis (see Ref. 22).

${ }^{\mathrm{b}} X_{A}=0.256$

${ }^{\mathrm{c}} \Delta H^{\mathrm{diss}}=4.02 \mathrm{kcal} / \mathrm{mol}$ (Ref. 29 ).

${ }^{\mathrm{d}}$ Reference 10 .

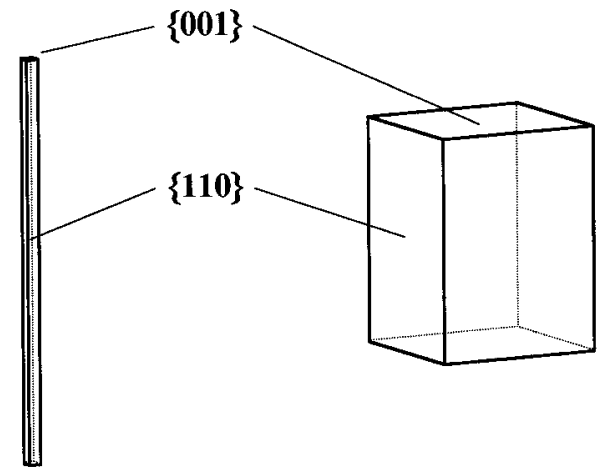

(a)

(b)

FIG. 5. Wulff constructions of the growth morphology of urea crystals based on different formalisms. (a) The growth morphology predicted according to the approaches in this study [see Eq. (7)]. (b) the growth morphology predicted according to the Hartman-Perdok theory (Refs. 3-5, 22), where it was assumed that $R_{h k l}^{\mathrm{rel}} \sim E_{h k l}^{\mathrm{att}} / E^{\mathrm{cr}}=\left(1-\xi_{h k l}\right)$ (see Table I for $\left.\xi_{h k l}\right)$.

The simulations shows that some degrees of ordering occur for the fluid molecules at the interfaces while solid urea molecules at the interfaces appear to have some degrees of disordering due to the relaxation. Snapshots along the interfaces of $\{001\}$ and $\{110\}$ are shown in Fig. 3. The urea molecules at the (110) interface are much more randomly distributed and oriented than those at $\{001\}$ faces, giving rise to an increased interfacial entropy. Although the degree of ordering of the interfacial liquid appears to its full extent from statistical averages of the generated coordinates, a good impression of the differences between the two interfaces can already be obtained from these snapshots.

By averaging the time series of the generated coordinates, density profiles and orientational distributions of the adsorbed solute urea molecules at the interface were calculated. In Fig. 4(a), orientational distributions of the dipole vectors $(\mathrm{O} \rightarrow \mathrm{C})$ of solute urea at the surface of $(001)$ and (110) are shown as a function of $\cos \theta(\theta$ is the angle between the $\mathrm{O} \rightarrow \mathrm{C}$ vectors and the outward surface normal). This curve is then converted to the $G^{*}(\theta)$ curve according to Eq. (20a). [See Fig. 4(b).]

In accordance with the above definition, $F_{1}$ urea molecules in the first fluid layer at the $\{001\}$ surfaces should have their $\mathrm{O} \rightarrow \mathrm{C}$ dipoles roughly anti parallel to the outward surface normal $\left(\theta \sim 180^{\circ}\right)$ [see Fig. 3(a)]. In Fig. 4(b), a prominent minimum occurs at $\theta \approx 180^{\circ}$. This implies that fluid urea units are ordered at the interface such that many $F_{1}$-like molecules occur. Apart from this minimum, the transformation from other orientations to the orientation of $\theta \approx 180$ may need to pass at most the small potential barrier $\Delta G_{(001)}^{*}\left(\leqslant k_{b} T\right)$. It can be roughly estimated ${ }^{6}$ that $\Delta G_{\text {kink }}^{*} \sim k_{b} T$. This suggests that $\Delta G_{\text {kink }}^{*}>\Delta G_{(001)}^{*}$, meaning that a case similar to case (I) occurs. In addition to the $\mathrm{O} \rightarrow \mathrm{C}$ distributions, we should also look at the distributions of $N-N$ vector of urea molecules. An analysis as given above leads to the same conclusion for the $\{001\}$ faces. It then follows from our calculations that almost all solute urea molecules can be regarded as effective growth units $(\delta \approx 1)$. 


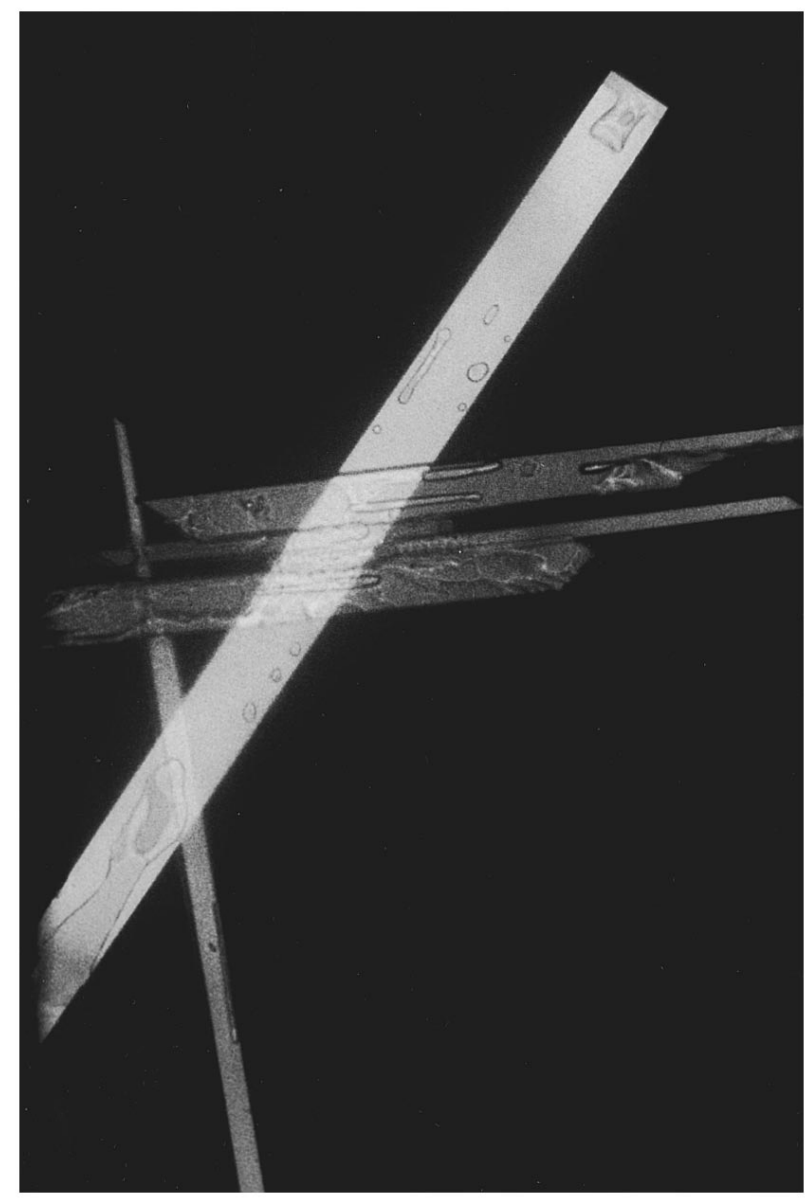

FIG. 6. Urea crystals grown from a aqueous solution (the equilibrium temperature $T_{s}=41.45^{\circ} \mathrm{C}$, and the supercooling $\left.\Delta T=0.35^{\circ} \mathrm{C}\right)$. The crystals have a long needle shape.

Unlike the $\{001\}$ faces, $F_{1}$-like urea molecules at the $\{110\}$ faces should have their $\mathrm{O} \rightarrow \mathrm{C}$ dipoles roughly parallel to the surface $\left(\theta \sim 90^{\circ}\right)$ [see Fig. 2(b)]. It is shown in Fig. 4(b) that $\Delta G_{(110)}^{*} \geqslant 2 \Delta G_{(001)}^{*}$. In this case urea molecules with $60^{\circ} \leqslant \theta \leqslant 120^{\circ}$ are regarded as $F_{1}$-like units. [It can be seen from Fig. 4(b) that $\Delta G^{*}$ of those molecules are equal to or less than zero.] To calculate $X_{A(h k l)}^{\text {eff }}$ Eq. (21) should be applied. (The same analysis for the $N-N$ distributions is also carried out for these faces.) Substituting $X_{A(h k l)}^{\text {eff }}$ and $X_{A}$ into Eq. (6) yields $C_{\ell(h k l)}^{*}$. We summarize in Table I the results, together with parameters $n_{h k l}, \xi_{h k l}$, and $d_{h k l}$.

Based on these data, $R_{h k l}^{\text {rel }}$ is obtained for the two orientations applying Eq. (7) (see the table for the results). We then construct the growth form of urea crystals using the Wulff plots. ${ }^{3,4,7}$ It follows that in case of growth from aqueous solutions, urea crystals obtain a needlelike shape. [See Fig. 5(a)]. On the other hand, a block shape of urea crystals was predicted applying the conventional Hartman-Perdok theory $^{23}$ [see Fig. 5(b)].

The growth morphology of urea crystals grown from aqueous solutions has been observed by other authors ${ }^{21,22}$ and also by ourselves. The results show that urea crystals indeed have a long needlelike shape, bounded by the $\{110\}$ and the $\{001\}$ faces (sometimes also the $\{111\}$ faces) (see Fig. $6)$. In addition, the ratio between the growth rate of the $\{110\}$ faces and that of the $\{001\}$ faces turns out to be almost 1:50 from experiments. ${ }^{21}$ Table I shows that our approach leads to a ratio of 1:35 while the Hartman-Perdok theory results in a value of $1: 1.4$. Obviously, our result is in good agreement with experiments.

\section{DISCUSSION}

In Sec. III, one has seen the good agreement between our predicted and the observed morphology. We have also applied the similar approach to predict the morphology of triclinic and orthorhombic $n$-alkane crystals, $\mathrm{C}_{60}$ crystals and other crystals. In each case, the results are in excellent agreement with observations. (The results are to be published elsewhere.) According to our research, once a PBC analysis and an IS analysis are carried out properly, the results obtained from our formalism can always be one of the best for the prediction of the growth morphology of crystals. This is attributed to the refined and more precise relation between $R_{h k l}^{\text {rel }}$ and the habit-controlling factors [Eq. (7)], and the estimation of the external habit-controlling factor $C_{\ell(h k l)}^{*}$ by the solid-fluid interfacial structure analysis. As can be seen from the discussions in the previous sections, the subtle influence of the fluid phase on the growth and the morphology of crystals can be examined by the IS analysis. This influence actually results from the change of the solid-fluid structure caused by the fluid units. It follows from our recent calculations $^{13,14}$ that any change in the concentration and the composition of the solution will subsequently alter the density and the orientational distribution of growth units at the crystal surface. This will, according to Eq. (21), change $X_{A(h k l)}^{\text {eff }}\left[\right.$ then $\left.C_{\ell(h k l) i}^{*}\right]$, and exert an influence on the morphology of crystals.

It is noted that to modify the morphology of crystals becomes more and more important for industries. For a given crystal structure, this can only be done by changing the external habit-controlling factors, especially $C_{\ell(h k l)}^{*}$. In order to increase the area of a crystal face, one needs to inhibit the growth of this face. This can be fulfilled by increasing the $C_{\ell(h k l)}^{*}$ value of this face. From this point of view, $C_{\ell(h k l)}^{*}$ can be used to measure the inhibition efficiency for the growth of the crystal faces. For a given crystallographic orientation, a high $C_{\ell(h k l)}^{*}$ will actually lead to a slow growth rate and a high inhibition efficiency.

In order to obtain a higher $C_{\ell(h k l)}^{*}$ for a crystal surface, we can try to decrease $X_{A(h k l)}^{\text {eff }}\left(\right.$ not $\left.X_{A}\right)$. Normally, if the solvent or an impurity is very strongly adsorbed at the crystal surface and the interfacial structure will be severally disturbed. This will cause a substantial reduction of $X_{A(h k l)}$ and $X_{A(h k l)}^{\mathrm{eff}}$, and result in an increase in $C_{\ell(h k l)}^{*}$ [see Eq. (6)].

In conclusion, we have analyzed the relation between the growth habit of crystals and the habit-controlling factors, especially the external factor $C_{\ell(h k l)}^{*}$. In order to predict properly the growth morphology of crystals, the morphological analysis, including both the interfacial structure (IS) analysis and the conventional PBC analysis, should be carried out. For solutions of complex molecules, the IS analysis can be summarized as the following steps: (a) calculate or determine the solid-fluid structure (including the orientation of 
solid units, and the density distributions and the orientational distributions of fluid molecules at the crystal surface); (b) identify $F_{1}$ growth units and calculate the concentration of effective growth units. This analysis will result in $C_{\ell(h k l)}^{*}$ for different crystallographic orientations. It follows that the growth morphology of crystals can finally be predicted by substituting this factor and those derived from the PBC analysis into an established formula like Eq. (7).

${ }^{1}$ N. Steno, De Solido Intra Solidum Naturaliter Contento Dissertationis Prodromus (Florence, 1669).

${ }^{2}$ J. D. H. Donnay and D. Harker, Am. Mineral. 22, 446 (1937).

${ }^{3}$ P. Hartman, in Morphology of Crystals, edited by I. Sunagawa (Terra, Tokyo, 1987), p. 269.

${ }^{4}$ P. Bennema, and J. P. van der Eerden, in Morphology of Crystals, Part A, edited by I. Sunagawa (Terra Sci., Tokyo, 1987), p. 1.

${ }^{5}$ P. Bennema, in Handbook on Cryst. Growth, edited by D. T. J. Hurle (North-Holland, Amsterdam, 1993), p. 477.

${ }^{6}$ A. A. Chernov, Modern Crystallography III-Crystal Growth, 1 (Springer, Berlin, 1984).

${ }^{7}$ P. Bennema and G. H. Gilmer, in Crystals Growth: An Introduction, edited by P. Hartman (North Holland, Amsterdam, 1973), p. 263.

${ }^{8}$ X. Y. Liu and P. Bennema, Phys. Rev. B 49, 765 (1994).

${ }^{9}$ X. Y. Liu and P. Bennema, Phys. Rev. B (to be published).

${ }^{10}$ X. Y. Liu and P. Bennema, J. Chem. Phys. 98, 5863 (1993).

${ }^{11}$ X. Y. Liu and P. Bennema, Phys. Rev. E 48, 2006 (1993).
${ }^{12}$ X. Y. Liu, Phys. Rev. E 49, 583 (1994).

${ }^{13}$ X. Y. Liu, Surf. Sci. 290, 403 (1993).

${ }^{14}$ S. R. Fowler and E. A. Guggenhein, Statistical Thermodynamics (Cambridge University, London, 1960).

${ }^{15}$ F. F. Abraham and J. Q. Broughton, Phys. Rev. Lett. 56, 734 (1986).

${ }^{16}$ A. D. Haymet and D. W. Oxtoby, J. Chem. Phys. 74, 2559 (1981).

${ }^{17}$ J. Q. Broughton and G. H. Gilmer, J. Chem. Phys. 84, 5741 (1986); 84, 5749 (1986); 84, 5759 (1986)

${ }^{18}$ E. S. Boek, W. J. Briels, J. van Eerden, and D. Feil, J. Chem. Phys. 96, 7010 (1992).

${ }^{19}$ E. S. Boek, W. J. Briels, and D. Feil, J. Phys. Chem. 98, 1674 (1994).

${ }^{20}$ J. E. Worsham, J. H. A. Levy, and S. W. Peterson, Acta Crystallogr. 10, 319 (1957).

${ }^{21}$ R. Davey, W. Fila, and J. Garside, J. Cryst. Growth 79, 607 (1986).

${ }^{22}$ R. Docherty, K. J. Roberts, V. Saunders, S. Black, and R. J. Davey, Faraday Discuss. 95, 11 (1993).

${ }^{23}$ E. S. Boek, D. Feil, W. J. Briels, and P. Bennema, J. Cryst. Growth 114, 389 (1991).

${ }^{24}$ W. F. van Gunsteren and H. J. C. Berendsen, Groningen Molecular Simulation (GROMOS) Library, Biomos, Groningen, 1987.

${ }^{25}$ A. T. Hagler, E. Huler, and S. Lifson, J. Am. Chem. Soc. 96, 5319 (1976).

${ }^{26}$ J. Hermans, H. J. C. Berendsen, W. F. van Cunsteren, and J. P. M. Postma, Biopolymers 23, 1513 (1984).

${ }^{27}$ H. J. C. Berendsen, J. R. Grigera, and T. P. Straatsma, J. Phys. Chem. 91, 6269 (1987).

${ }^{28}$ W. F. van Gunsteren and H. J. C. Berendsen, Mol. Phys. 34, 1311 (1977).

${ }^{29}$ M. Pickering, J. Chem. Ed. 64, 723 (1987). 